\title{
Polyvinyl Alcohol (PVA) Partially Hydrolyzed Addition in Synthesis of Natural Hydrogel Carboxymethyl Cellulose (CMC) Based from Water Hyacinth
}

\author{
Asep Handaya Saputra ${ }^{1, *}$, Nadia Huda Apriliana ${ }^{2}$ \\ ${ }^{1}$ Department of Chemical Engineering, Faculty of Engineering Universitas Indonesia Depok 16424 \\ ${ }^{2}$ Department of Chemical Engineering, Faculty of Engineering Universitas Indonesia Depok 16424
}

\begin{abstract}
There are many benefits from Indonesia's biodiversity. One of them is cellulose of Water hyacinth. Water hyacinth contains $60 \%$ cellulose. Cellulose of water hyacinth can be used as material for polymer product such as hydrogel. Hydrogel is a hydrophilic superabsorbent polymer that can absorb water until 200 times from its weight without dissolved in water. In synthesis of hydrogel, the cellulose should be modified to Carboxymethyl Cellulose (CMC) by alkalization and carboxymethylation process in reaction media ethanol and isopropanol (2:8). The aim of the study is to obtain the hydrogel with high swelling ratio characteristic by adding Polyvinyl Alcohol (PVA) Partially Hydrolyzed with variation of PVA/CMC composition (20:80, $80: 20,50: 50,25: 75$ and 75:25). The optimum PVA/CMC composition are 20:80 and 25:75 which give results $29 \%$ and $25 \%$ in their swelling ratio. This low swelling ratio is caused by morphology structure of hydrogel that has a compact structure and small pores. This structures lead to difficulity in water diffusion. In compare with hydrogel from PVA Fully Hydrolyzed addition, hydrogel with PVA Partially Hydrolyzed addition contain vinyl acetate that cause hydrogel less hydrophilic and has a low value on liofil-hidrophilic balance.
\end{abstract}

\section{Introduction}

The amount of biodiversity in Indonesia has became a great opportunity to explore its potential. For example, water hyacinth can be utilized to obtain natural polymer in cellulose form. Water hyacinth consists of $60 \%$ cellulose, $8 \%$ hemicellulose, and $17 \%$ lignin $^{[1]}$. Utilization of water hyacinth may enhanced its value, since water hyacinth is noxious weed due to its congested growth.

Fiber of water hyacinth can be used as the basic material of polymer product in the form of hydrogel. Hydrogel is a superabsorbent polymer with the ability to absorb liquid up to 200 times the weight of itself without getting dissolved in water. This polymer product is environmental friendly for it easy to be dissolved.

Hydrogel can be used in many applications. Recently, extensive research and development has been conducted for hydrogel applications in the scopes of healthcare, biomedicine, agriculture, pharmacy, food packaging, horticulture industry and other scopes. As in healthcare, hydrogel can be used as wound dressing, drug release system, contact lenses, and transdermal drug delivery. In agriculture sector, hydrogel can ensure the availability of water, reduce the frequency of irrigation up to $50 \%$ and also applicable to areas that lacks of water supplies. Futhermore, hydrogel can be used as constituent material in diaper and period pads.

Hydrogel is classified into two categories, the natural and the synthetic hydrogel. Hydrogel from water hyacinth cellulose is classified into natural hydrogel, whereas synthetic hydrogel is Polyhydroxyethyl Methacrylate (pHEMA) dan Polyacrylamide. Synthetic hydrogel is not biodegradable and may increase air pollution, since about $90 \%$ of used hydrogel ought to be incinerated or disposed on landfills. ${ }^{[2]}$

In synthesis of hydrogel, cellulose needs to be modified to become its derivative, Carboxylmethyl Cellulose (CMC), for it has a functional group which causes electrostatic repulsion, that would arise between macromolecules, and water would be taken up. ${ }^{[3]}$

Moreover, addition of hydrophilic polymer: Polyvinyl Alcohol (PVA) is essential, in order to maximize water absorption. PVA is not only biodegradable and nontoxic, but also has stable both thermal and chemical properties. PVA is classified into 2 groups based on its synthesis process, PVA Partially Hydrolyzed and PVA Fully Hydrolyzed.

PVA fully hydrolyzed has a high degree of crystallinity. Addition of fully hydrolyzed PVA results in

Corresponding author: nadia.huda@ui.ac.id 
reduction of swelling ratio as reported in previous research that was conducted in the same laboratory and using the same instrument. ${ }^{[4]}$

Polymer with high crytallinity embodies the characteristic of being physically stable. Consequently, it is difficult for water as a swelling medium to enter within the structure. ${ }^{[5]}$ Hence, the synthesis of hydrogel in this study will add PVA Partially Hydrolyzed which has low value in degree of crystallinity. Addition of PVA Partially hydrolyzed is expected to produce hydrogel with higher swelling ratio properties.

The purpose of this study is to obtain optimum composition ratio of PVA and CMC in hydrogel synthesis, based on the properties and characteristics of hydrogel. Addition of PVA Partially Hydrolyzed and its effects on properties and characteristics of produced hydrogel were studied. Differences between addition of PVA Partially Hydrolyzed and PVA Fully Hydrolyzed on synthesis hydrogel were also analysed.

\section{Materials and Methods}

\subsection{Material}

This research used water hyacinth from Cipondoh Lake, Tangerang, Indonesia. Several materials that used for isolating cellulose are toluene, ethanol, $\mathrm{NaClO}_{2}, \mathrm{NaOH}$, distilled water, acetate acid, and buffer solution. While materials that used for synthesis $\mathrm{CMC}$ and hydrogel are $\mathrm{Na}-\mathrm{MCA}, \mathrm{NaOH}$, ethanol, isopropanol, and PVA Partially Hydrolyzed.

\subsection{Methods}

\subsubsection{Isolation of Water Hyacinth Cellulose}

Preparation started with separation of water hyacinth stems (part with highest cellulose content) from roots and leaves. Stem was dried under direct sunlight for 7 days to remove water content. The dried stem was grinded and sieved with Willey Ball Mill until it reached size of 60 mesh. The next step was dewaxing. The water hyacinth powder was wrapped in filter paper, then extracted with Soxhlet Apparatus and mixture of toluene and ethanol (2:1) as solvent.

Prior to lignin content removal, the water hyacinth powder was mixed with $1 \%$ wt solution of $\mathrm{NaClO}_{2}$. The mixture was put into prepared bottles and laid in water bath at $80^{\circ} \mathrm{C}$ for 3 hours. After lignin removal process, the powder was soaked in $\mathrm{NaOH} 17.5 \%$ solutions for 3 hours to remove hemicellulose. The powder was washed and neutralized with dillistilate water or $\mathrm{CH}_{3} \mathrm{COOH}$ and dried in room temperature. This isolation obtained $\alpha$ cellulose from water hyacinth.

\subsubsection{Synthesis of Carboxymethyl Cellulose (CMC)}

Synthesis of Carboxymethyl Cellulose (CMC) consists of alkalization and carboxymethylation process. Alkalization process was conducted by mixing 5 grams cellulose powder that obtained from isolation cellulose process with ethanol and isopropanol $(2: 8)$ as reaction medium. Then, the solution was put in 3 neck flash with stirrer for 10 minutes and then added with $20 \mathrm{ml}$ of $10 \%$ of $\mathrm{NaOH}$. The alkalization process was spent 1 hour at room temperature. The next step was carboxymethylation process which conducted by adding NaMCA as much as 6 grams and stirred it for 3.5 hours at $55{ }^{\circ} \mathrm{C} .^{[6]}$ The obtained $\mathrm{CMC}$ was neutralized and purified with $\mathrm{CH}_{3} \mathrm{COOH}$ and $96 \%$ ethanol then dried for one night at room temperature. To determine the degree of substitution, solid CMC was analyzed in BPPK Bandung.

\subsubsection{Synthesis of Hydrogel}

Synthesis of hydrogel was initiated by mixing CMC and Polyvinyl Alcohol (PVA) Partially Hydrolyed with various compositions $(20: 80,80: 20,50: 50,25: 75$ and $75: 25)$. The solution was mixed for 10 minutes at room temperature. Then the esterification process was conducted by adding $10 \%$ citric acid ${ }^{[7]}$ and stirred it for 2 hours until it was homogeneous.

The hydrogel solution was put on petri dish to mould the hydrogel with a thickness of $10 \mathrm{~mm}$ and a diameter of $90 \mathrm{~mm}$. Next, Hydrogel solution was pre-dried for 24 hours at $30^{\circ} \mathrm{C}$ at vacuum condition, followed by drying process of hydrogel. Hydrogel solution dried in oven at $80^{\circ} \mathrm{C}$ for 13 hours. The procedure above was repeated with different composition of PVA and CMC.

The hydrogel was analyzed with FTIR, SEM, and swelling ratio to obtain the characteristics of hydrogel. To obtain the swelling ratio, each sample was weighed before and after soaked in distilled water for 1 hour at room temperature. After that, the excess of distilled water on the surface of the hydrogel is removed with filter paper (blotting) and the hydrogel after immersion was weighed. ${ }^{[8]}$ Measurement of swelling ratio can be determined by Eq. (1) :

$$
\text { Swelling Ratio }=\frac{W_{L}-W_{0}}{W_{o}} \times 100 \%
$$

\section{Result and Discussion}

\subsection{Water Hyacinth $\alpha$-cellulose}

Dewaxing process is a process to remove wax, pectin, and chlorophyll. As a result of dewaxing process, solvent and filter paper show marked colour change. On the other hand, bleaching is lignin removal process that occurs due to bleaching reaction of Chlor Dioxide $\left(\mathrm{ClO}_{2}\right)$ in acid media as shown in reaction below.

$$
\begin{aligned}
\mathrm{NaClO}_{2} & +\mathrm{CH}_{3} \mathrm{COOH} \rightarrow \mathrm{ClO}_{2}+\mathrm{NaHCO}_{2}+\mathrm{CH}_{3} \\
\mathrm{ClO}_{2} & \text { lignin } \rightarrow \text { oxidized lignin }+\mathrm{HClO}_{3}+\mathrm{HClO}_{2}
\end{aligned}
$$

Bleaching solution will show a colour change to yellow, owing to the presence of oxidized lignin. This colour change indicatied lignin removal from powder, 
and caused the colour of the powder change into white. After dehemicellulose process, a pure $\alpha$-cellulose was obtained and it has white colour powder.

\subsection{Carboxymethyl Cellulose (CMC)}

Synthesis of Carboxymethyl Cellulose (CMC) started with alkalization process then carboxymethylation process. The process occurs in reaction media of ethanol and isopropanol $(2: 8)$. Polarity of reaction media plays significant role in alkalization process. Reaction media solvent with low polarity will increases reactivity of alkalization reaction, since $\mathrm{NaOH}$ will be more reactive to cellulose compared to the reaction medium. Besides the higher concentration of $\mathrm{NaOH}$ that exceeds its optimum concentration will cause $\mathrm{NaOH}$ more soluble and difficult to substitute - $\mathrm{OH}$ group in cellulose as a result cellulose has crystalline structure. The crystalline structure also cause carboxymethylation process occurs hardly. Due to the cellulose still contain the lignin, CMC powder has yellow colour as shown in Figure 1.

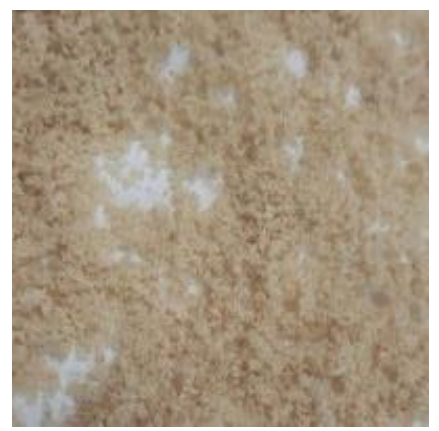

Fig. 1. Carboxymethyl Cellulose (CMC)

The successful synthesis CMC can be indicated by measuring the degree of substitution (DS) that conducted in BPPK Bandung. The result of DS value in this study is 1.64 of 3 (maximum DS value of CMC). This result has relevant value with previous study which uses $\mathrm{NaOH}$ $10 \%$ and has DS value 1.65 and another study which uses same reaction media has 1.59 of DS value.

\subsection{Hydrogel}

The hydrogel synthesis in this study utilizes CMC as the main material and PVA Partially Hydrolyzed as the additive. The hydrogel synthesis method employed the chemical crosslinking with citric acid monohydrate and carboxylic group as the cross linker agent. ${ }^{[7]}$ Table 1 shows the variation of the composition between CMC and PVA Partially Hydrolyzed.

Table 1. The Variation of The Composition between CMC and PVA Partially Hydrolyzed

\begin{tabular}{|c|c|c|c|}
\hline \multicolumn{3}{|c|}{ PVA : CMC Composition } & Sample \\
\hline $50: 50$ & $2: 2$ & 2 grams $: 2$ grams & P5C5 \\
\hline $25: 75$ & $1: 3$ & 1 grams $: 3$ grams & P1C3 \\
\hline $75: 25$ & $3: 1$ & 3 grams $:$ grams & P3C1 \\
\hline $80: 20$ & $4: 1$ & 3.2 grams $: 0.8$ grams & P4C1 \\
\hline $20: 80$ & $1: 4$ & 0.8 grams $: 3.2$ grams & P1C4 \\
\hline
\end{tabular}

Both polymers are mixed and stirred with $10 \%$ citric acid, and results in homogenous hydrogel solution. Then, the solution undergoes two drying processes, the predrier process inside vacuum oven for 24 hours and drying process at $80^{\circ} \mathrm{C}$ for 13 hours. The process of predrier aims at cyclic anhydra formation in the citric acid, while the final drying process of hydrogel causes the hydrogel bonds in the hydroxyl group in each of the PVA and CMC to lose, so that both polymers can be connected in the hydrogel structure. This drying process aligns to the reaction mechanism as in Figure 2.<smiles>CC(=O)OCC(=O)OC1(O)CC(=O)OC1CC(=O)O</smiles><smiles>CCCC(C)(CC(=O)OC)C(=O)OC</smiles><smiles>C=CC[C@](C)(CC)CC(=O)OC</smiles><smiles>CCCCC(C)(C)CC(=O)OC</smiles>

Fig. 2. The Reaction Mechanism of Synthesis Hydrogel

From pictures shown in table 2, it can be concluded that increasing PVA compositions, such as the P3C1type hydrogel (PVA :CMC 75:25) and P4C1 (PVA:CMC 80:20) will results in more adhesive and soft hydrogels.

This results may be caused by the growth of the number pof acetate group with thermoplastic properties. Thermoplastic property is prone to melting or soften when being heated or cooled as well as is prone to giving rubbery property. ${ }^{[10]}$

Hence, in synthesis of hydrogel, the optimum composition ratio of PVA and CMC were 20:80 (Hydrogel P1C4) and 25: 75 (Hydrogel P1C3). Assynthesized hydrogels are generated in sheet form, ransparant, and non-adhesive. 
Table 2. Hydrogels with Different Composition

\begin{tabular}{|c|c|}
\hline P1C4 (PVA:CMC 20 : 80) & P1C3 (PVA:CMC 25:75) \\
\hline & \\
\hline & \\
\hline & \\
\hline & \\
\hline & \\
\hline
\end{tabular}

\subsection{Hydrogel, PVA Partially Hydrolyzed, and CMC FTIR Analysis}

CMC, PVA Partially Hydrolyzed, and Hydrogel P1C3 samples were analyzed by FTIR, in order to determine the functional group content. Figure 3 shows FTIR spectra image of CMC. The functional groups formed out of CMC are hydroxyl group (-OH), ether group (-O), and carboxyl group (-COO). In CMC, there is a peak in the wave of $1589.17 \mathrm{~cm}^{-1}$ which indicates the presence of aromatic group. That occurs because the presence of lignin content in the cellulose which has a polymer structure of aromatic group. The presence of lignin content is also supported by the yellow color produced by CMC.

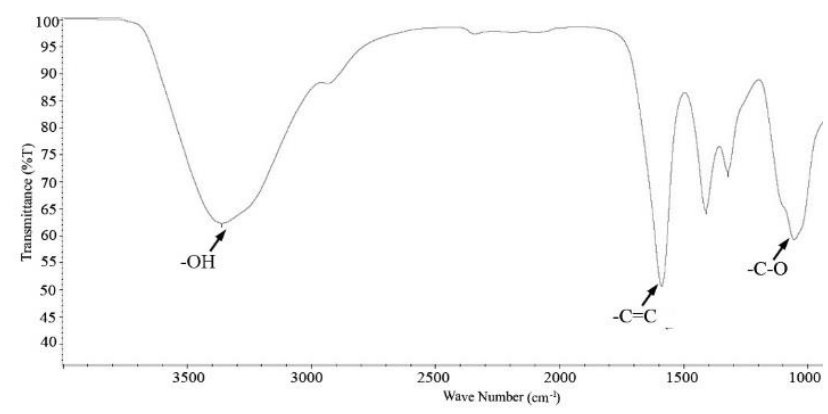

Fig. 3. FTIR spectra of CMC

Figure 4 is the FTIR spectra image on the sample of PVA Partially Hydrolyzed. The functional groups in PVA are hydroxyl group, carboxyl group, and ether group. Carboxyl and ether groups arise due to the fact that the PVA used is the PVA Partially Hydrolyzed, so there are still carboxyl group that has not hydrolyzed into hydroxyl group and still being present in the vinyl acetate.

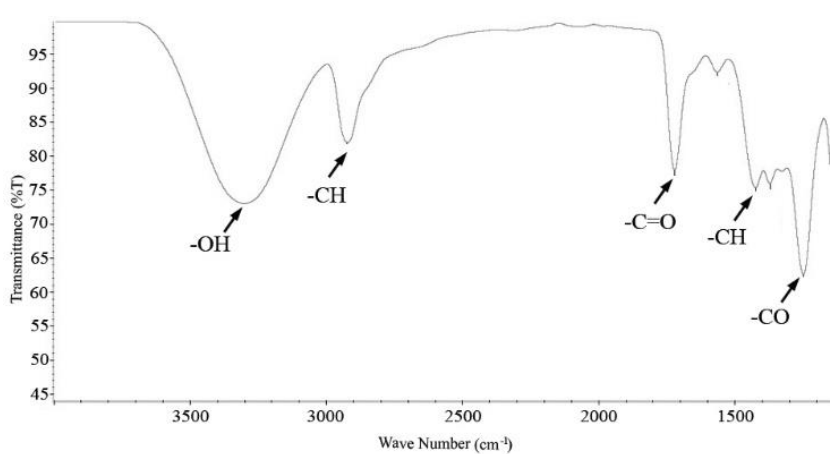

Figure. 4. FTIR spectra of PVA Partially Hydrolyzed

The FTIR spectra of the hydrogel is shown in Figure 5. The hydrogel used is $\mathrm{P} 1 \mathrm{C} 3$ with the composition of PVA:CMC 25:75. The hydrogel has hydrophilic group in the form of hydroxyl group, alkane group, and -CO group which show that the formed hydrogel contains ester group of PVA Partially Hydrolyzed. The success of synthesis hydrogel can be seen in the wave number of $1716 \mathrm{~cm}^{-1}$ which indicates the presence of carboxyl group. $^{[7]}$ The presence of such group signifies a crosslinking reaction which occurs between PVA Partially Hydrolyzed, citric acid, and CMC in order to form hydrogel tissue.

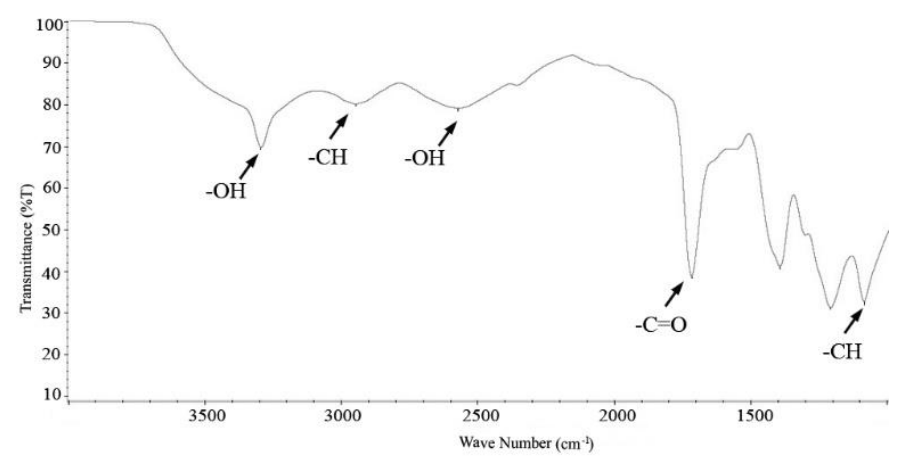

Fig. 5. FTIR Spectra of Hydrogel

The FTIR spectra of CMC, PVA, and hydrogel are presented in Figure 6. From the graph, it can be seen regarding the different transmittance of hydroxyl group. Hydroxyl group (-OH) in $\mathrm{CMC}$ has the lowest transmittance value followed by PVA and Hydrogel. This is supported by the fact that formed hydrogel consists of larger composition of CMC compared to the composition of PVA. The transmittance value of hydrogel is the lowest and this shows that the addition of PVA Partially Hydrolyzed does not increase the hydroxyl group content in the hydrogel. 


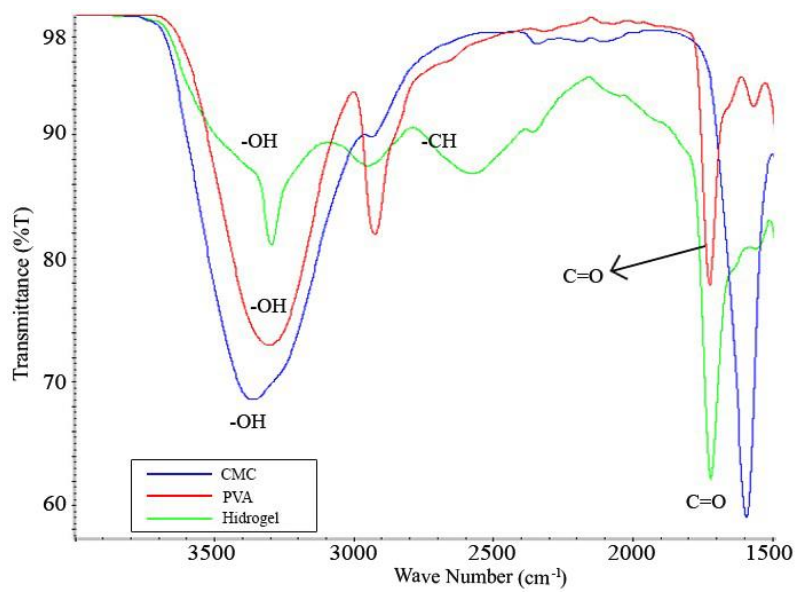

Fig. 6. The FTIR Spectra of CMC, PVA Partially Hydrolyzed, and Hydrogel

\subsection{Hydrogel, PVA Partially Hydrolyzed, and CMC Swelling Ratio Analysis}

Hydrogel polymers content will have significant role in determining hydrogel swelling ratio. Table 3 displays that the addition of PVA Partially Hydrolyzed decreases the swelling ratio of the hydrogel.

Table 3. Swelling Ratio of Hydrogel

\begin{tabular}{|c|c|c|}
\hline $\begin{array}{c}\text { Hydrogel } \\
\text { types }\end{array}$ & $\begin{array}{c}\text { PVA:CMC } \\
\text { Composition }\end{array}$ & $\begin{array}{c}\text { Swelling Ratio } \\
\text { Value (\%) }\end{array}$ \\
\hline P1C4 & $20: 80$ & 29,22 \\
\hline P1C3 & $25: 75$ & 25,75 \\
\hline
\end{tabular}

Meanwhile, higher composition of CMC results in higher swelling ratio value. Being associated with the result of FTIR analysis, PVA Partially Hydrolyzed has fewer hydroxyl group than CMC. Hence, this may decrease the hydrophilic characteristic of the hydrogel because PVA Partially Hydrolyzed is not being more hydrophilic than CMC. ${ }^{[11]}$ Additionally, the addition of PVA reduces the double role of swelling in CMC-based hydrogel, as well. The aforementioned swelling is the opening of a hydrogel tissue which allows water to enter. Such swelling occurs due to electrostatic repulsion by a protonated carboxyl group (COO-) of $\mathrm{CMC}^{[3]}$ The highest swelling value is achieved by $\mathrm{P} 1 \mathrm{C} 4$ with the composition of PVA:CMC 20:80 and the average selling of $29 \%$, while P1C3 has the composition of PVA:CMC 25:72 and the average selling of $25 \%$.

Low swelling ratio of hydrogel may be induced by the surface morphology of the hydrogel. From Figure 7, it can be seen that P1C4 has larger pores and is less compact in terms of structure. Thus, swelling ratio of this hydrogel is higher than P1C3. Pores have a role in water absorption of hydrogel. ${ }^{[12]}$ Compact surface morphology will act as a barrier to water diffusion.

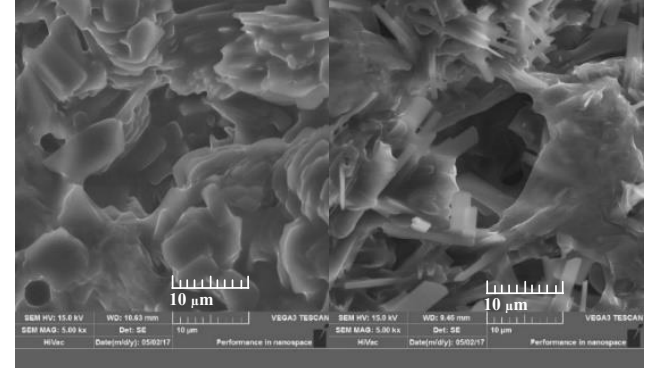

Fig. 7. Microscopic surface of a) Hydrogel $\mathrm{P} 1 \mathrm{C} 3$ and b) Hydrogel P1C4

Hence, Hydrogels P1C4 and P1C3 have swelling ratio characteristics $29 \%$ and $25 \%$, respectively. Low swelling ratio might be originated from more compact morphology structure and less pores. Consequently, this condition acts as barrier to water diffusion. The characteristics of functional groups in PVA Partially Hydrolyzed hydrogels is characterized by the presence of hydroxyl group (-OH) as water absorbent group and carboxyl group $(\mathrm{C}=\mathrm{O})$ as an indication of hydrogel successful crosslinking reaction.

\subsection{The Comparison of Hydrogel-PVA Fully Hydrolyzed and Hydrogel PVA-Partially Hydrolyzed}

The comparison between hydrogel-PVA Fully Hydrolyzed and hydrogel-PVA Partially Hydrolyzed is based on the FTIR analysis and the morphological structure. Based on the FTIR result between Partially Hydrolyzed PVA and Fully Hydrolyzed PVA, the differences are visible in the transmittance values of $\mathrm{C}-\mathrm{O}$ and $\mathrm{C}=\mathrm{O}$ groups. These results imply that the hydrogel derived from PVA Partially Hydrolyzed with the content of vinyl acetate having not been hydrolyzed.

The hydroxyl group in the PVA has hydrophilic properties, while the acetate group is a hydrophobic. Polymers with hydrophobic properties show a low affinity towards water. $^{[14]}$ In addition, the presence of acetate group residue weakens the intra and intermolecular hydrogen bonds from the adjacent hydroxyl group. The hydrogen bond with different molecule is the bond which occurs between hydrogel and water molecule. One of the factors in determining the swelling properties of hydrogel is also the hydrophiliclipophilic balance (HLB) in the hydrogel. HLB is a function of the amount of functional group, side chain, and ions. Functional group enhancing the hydrophilic properties of gels is polar groups which are ionic and non-ionic at the same time. ${ }^{[15]}$ The ester group (carboxylic group) derived from PVA Partially Hydrolyzed on the hydrogel has low polarity compared to alcohol group $(-\mathrm{OH})$. The comparison of morphological structure is displayed in Figure 8. The hydrogel with the addition of PVA Partially Hydrolyzed has fewer and smaller pores than the hydrogel containing PVA Fully Hydrolyzed. This result confirms the fact that the acetate group affects PVA so that it is prone to having crystal-like structure (solid and tidy) when the PVA is heated. ${ }^{[16]}$ 


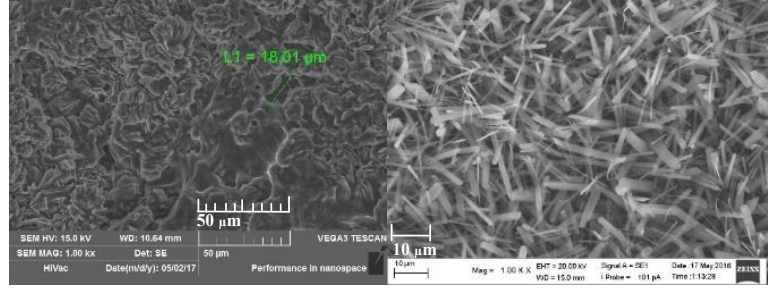

Figure. 8. Microscopic surface of a) Hydrogel- PVA Partially Hydrolyzed and b) Hydrogel-PVA Fully Hydrolyzed

Therefore, Hydrogel with the addition of PVA Partially Hydrolyzed has lower swelling ratio than hydrogel with PVA Fully Hydrolyzed, due to the presence of vinyl acetate.

\section{Conclusion}

This study showed that hydrogel can be produced from CMC and PVA partially hydrolyzed. The optimum composition ratio of PVA and CMC were 20:80 (Hydrogel P1C4) and 25 : 75 (Hydrogel P1C3). On the other hand, the swelling ratio of $\mathrm{P} 1 \mathrm{C} 4$ and $\mathrm{P} 1 \mathrm{C} 3$ are $29 \%$ and $25 \%$, respectively. Low swelling ratio might be originated from more compact morphology structure and less pores. Consequently, this condition acts as barrier to water diffusion. The characteristics of functional groups in PVA Partially Hydrolyzed hydrogels is characterized by the presence of hydroxyl group $(-\mathrm{OH})$ as water absorbent group and carboxyl group $(\mathrm{C}=\mathrm{O})$ as an indication of hydrogel successful crosslinking reaction. Hydrogel with the addition of PVA Partially Hydrolyzed has lower swelling ratio characteristic than hydrogel with PVA Fully Hydrolyzed, due to the presence of vinyl acetate.

\section{References}

1. A.F Abdel-Fattah and M. Abdel-Naby, Carbohydr. Polym., 87, 2109 (2012)

2. S. Kiatkamjornwong, K. Mongkolsawat and $M$. Sonsuk, Polym., 43, 3915 (2002)

3. R. Barbucci, A. Magnani, and M. Consumi, Macromol, 33, 7478 (2000)

4. E. Ogur, Materials, Processing and Applications, 16, 7 (2005)

5. K. Somwang, T. Enomae, A. Isogai, F. Onabe, Japan TAPPI J, 56, 866 (2002)

6. A.H Saputra, M. Hapsari, and A.B Pitaloka, Contemp. Eng. Sci., 8, 1573 (2015)

7. A.H Saputra, M. Hapsari, A.B Pitaloka, P.P.D.K Wulan, JESTECH, 2, 78 (2015)

8. J. Lu, Y. Li, D. Hu, X. Chen, Y. Liu, L. Wang, Y. Zhao, Biomed Res Int, 2015, 3 (2015)

9. B.K Barai, R.S Singhai and P.R. Kulkarni, Carbohyd. Polym., 32, 229 (1997)

10. A.J Kovacs, R.A Stratton, and J.D. Ferby, J. Phys. Chem., 67, 155 (2016)
11.G. Buhus, M. Popa, C. Peptu, J. Desbrieres, J. Optoelectron. Adv. M., 9, 3450 (2007)

12.M. Hashem, S. Sharaf, M.M Abd El-Hady, A. Hebeish, Carbohydr. Polym., 95, 425 (2013)

13.Z. Krol, M. Malik, K. Marycz, A. Jarmoluk, Polym., 8, 260 (2016)

14. M.L Bruschi, Strategies to Modify the Drug Release from Pharmaceutial Systems (Elsevier Ltd.) (2015)

15. N.C Dafader, S. Ganguli, M.A Sattar, M.E Haque, F.Akhtar, MPJ, 4, 37 (2009)

16.C.M Hassan and N.A Peppas, Polym. Sci,, 153, 38 (2000) 